\title{
Approximation of the Spectrum of Closed Operators: The Determination of Normal Modes of a Rotating Basin
}

\author{
By Jean Descloux, Mitchell Luskin* and Jacques Rappaz**
}

\begin{abstract}
This paper gives a theory of spectral approximation for closed operators in Banach spaces. The perturbation theory developed in this paper is applied to the study of a finite element procedure for approximating the spectral properties of a differential system modeling a fluid in a rotating basin.
\end{abstract}

Introduction. In this paper, we give a theory of spectral approximation for closed operators in Banach spaces. We then apply this theory to an analysis of the approximation of the spectral properties of some differential systems by finite element methods.

Bramble and Osborn [1] and Osborn [14] developed a theory of spectral approximation for compact operators in Banach spaces. Their theory can be applied to the analysis of many numerical procedures for the spectral approximation of differential operators, $T$, such that $T+\lambda I$ has a compact inverse for some $\lambda \in \mathbf{C}$. Most of the differential systems in the theory of elasticity are in this class.

However, there are many differential systems of interest in mathematical physics which do not have compact resolvents. These operators can have continuous spectrum, eigenvalues of infinite multiplicity, and finite limit points of eigenvalues. Also, the eigenfunctions need not be smooth since the differential systems are not necessarily elliptic.

Descloux, Nassif, and Rappaz [4], [5] have studied the approximation of the spectrum of a differential system of interest in magnetohydrodynamics which has a bounded inverse, but not a compact inverse. They developed a theory of spectral approximation for bounded operators which treats this problem. An analysis of the approximation of the spectral properties of a class of bounded operators by finite element methods has also been done by Mills [12], [13].

The results in this paper apply to closed (not necessarily bounded) operators in Banach spaces. We apply the perturbation theory developed in this paper to the study of a finite element procedure for approximating the spectral properties of a differential system modeling a fluid in a rotating basin. We note that, unlike previous authors, we analyze the approximation of the differential operator directly and not through its inverse.

Received January 3, 1980.

1980 Mathematics Subject Classification. Primary 65N25, 65N30.

*Supported by AFOSR under Contract F49620-79-C-0149 and by a Faculty Research Fellowship from the Horace Rackham School of Graduate Studies, The University of Michigan.

**Supported by the Fonds National Suisse de la Recherche Scientifique. 
The time-dependent equations for the differential system modeling a fluid in a rotating basin are

$$
\begin{gathered}
\frac{\partial \zeta}{\partial t}=-\nabla \cdot \vec{u}, \quad(x, t) \in \bar{\Omega} \times \mathbf{R}, \\
\frac{\partial \vec{u}}{\partial t}=-\nabla \zeta-f \vec{u}-\omega R \vec{u}, \quad(x, t) \in \bar{\Omega} \times \mathbf{R}, \\
\vec{u} \cdot \vec{n}=0, \quad(x, t) \in \partial \Omega \times \mathbf{R}, \\
\int_{\Omega} \zeta d x=0,
\end{gathered}
$$

where $\Omega \subseteq \mathbf{R}^{2}$ is a bounded, connected open set with smooth boundary, $\partial \Omega$, $\vec{u}=\left(u_{1}, u_{2}\right)$ is the horizontal volume transport, $\zeta$ is the height of the fluid above equilibrium level, $R$ is the linear operator $R \vec{u}=\left(-u_{2}, u_{1}\right), \vec{n}$ is the exterior normal to $\partial \Omega$, and $f \geqslant 0$ and $\omega$ are real constants representing friction and Coriolis terms.

Thus, we are concerned with approximating the spectral properties of the system

$$
\begin{array}{ll}
T(\zeta, \vec{u})=(-\nabla \cdot \vec{u},-\nabla \zeta-f \vec{u}-\omega R \vec{u}), & x \in \Omega, \\
\vec{u} \cdot \vec{n}=0, & x \in \partial \Omega, \\
\int_{\Omega} \zeta d x=0 . &
\end{array}
$$

We note that $T-I$ is a symmetric, formally dissipative operator with maximal nonpositive boundary conditions [9].

If $f=\omega=0$, then $T(0, R \nabla \psi)=0$ for all smooth functions $\psi$ such that $\psi=0$ on $\partial \Omega$. Hence, in this case $(f=\omega=0), 0$ is an eigenvalue of $T$ of infinite multiplicity. Since the sum of an operator with noncompact inverse and a bounded operator has a noncompact inverse, it follows that $T-\lambda I$ has a noncompact inverse for the general case $f, \omega \in \mathbf{R}, \lambda \in \mathbf{C}, \lambda$ belonging to the resolvent set of $T$.

We give here error estimates for a finite element procedure proposed by Platzman [15] to approximate the spectral properties of $(0.2)$. The selfadjoint case $f=0$ was analyzed by Luskin [11] by techniques different from those used here.

In Section 1, we give a general theory for the approximation of a closed operator $A$ by a family of finite-dimensional operators $\left\{A_{h}\right\}$. In our applications, $A$ will be a differential operator, and $A_{h}$ will be an approximation of $A$ given by a finite element procedure. We propose two properties, (P1) and (P2), and show that these properties imply the convergence of the spectral properties of $A_{h}$ to those of $A$. Error estimates in the applications will follow from Theorem 1.3.

We give special results in Section 2 for the case where $A$ and $A_{h}$ are selfadjoint operators in a Hilbert space. These results apply to the approximation of a continuous spectrum.

The operator theory developed in Sections 1 and 2 is applied, in Section 3 and 4, to examples of the approximation of spectral properties of differential operators by finite element methods. In Section 3, we apply our theory to obtain error estimates for the approximation of the spectral properties of scalar, second order, uniformly strongly elliptic operators by the standard finite element method. This example is included even though the results are not new since we believe that its inclusion will make it easier for the reader to understand our main example in Section 4.

In Section 4, we define and analyze the approximation of $T$. Theorem 1.3 gives 
optimal order estimates for the approximation of eigenspaces. Optimal order eigenvalue estimates for this problem have been given in [11] for the selfadjoint case and are derived from the results in this paper and in [3] for the general case.

We note that we use, without explicit reference, the classical spectral theory; see, for example, Kato [6], Riesz-Nagy [16].

1. Approximation of the Spectrum of Closed Operators in Banach Spaces. We first introduce some notation. Let $X$ be a complex Banach space with norm \|\| . Denote by $\mathscr{B}(X)$ the set of bounded, linear operators $B: X \rightarrow X$. Also, denote by $\mathcal{C}(X)$ the set of closed, linear operators $C: \mathscr{D}(C) \subset X \rightarrow X$, where the domain of $C, \mathscr{D}(C)$, is not necessarily dense in $X$. For $C \in \mathcal{C}(X), \rho(C)$ is the resolvent set of $C$ defined by

$$
\rho(C)=\left\{z \in \mathbf{C} \mid(z-C)^{-1} \in \mathscr{B}(X)\right\} .
$$

If $z \in \rho(C)$, we define the resolvent operator $R_{z}(C)=(z-C)^{-1}: X \rightarrow X$. The complement of $\rho(C)$ is $\sigma(C)=\{z \in \mathbf{C} \mid z \notin \rho(C)\}$, the spectrum of $C$.

Let $Y$ and $Z$ be closed subspaces of $X$ and $x \in X$. We set

$$
\begin{gathered}
\delta(x, Y)=\inf _{y \in Y}\|x-y\|, \quad \delta(Y, Z)=\sup _{\substack{y \in Y \\
\|y\|=1}} \delta(y, Z), \\
\hat{\delta}(Y, Z)=\max (\delta(Y, Z), \delta(Z, Y)) ;
\end{gathered}
$$

$\hat{\delta}(Y, Z)$ is called the gap between $Y$ and $Z$ and is a measure of the "distance" between these spaces. If $C$ and $D$ are in $\mathcal{C}(X)$, with graphs $G_{C}, G_{D} \subset X \times X$, then we define $\delta(C, D)=\delta\left(G_{C}, G_{D}\right)$, i.e.,

$$
\delta(C, D)=\sup _{\substack{x \in \mathcal{D}(C) \\\|x\|+\|C x\|=1}} \inf _{y \in \mathscr{D}(D)}\{\|x-y\|+\|C x-D y\|\} .
$$

Furthermore, we define

$$
\hat{\delta}(C, D)=\max (\delta(C, D), \delta(D, C))
$$

Finally, if $Y$ is a subspace of $\mathscr{D}(C) \cap \mathscr{D}(D)$, we set

$$
\|C-D\|_{Y}=\sup _{\substack{y \in Y \\\|y\|=1}}\|C y-D y\| .
$$

Now, let $A \in \mathcal{C}(X)$ be a given operator. In order to approximate $\sigma(A)$, we consider a family $\left\{X_{h}\right\}$ of finite-dimensional subspaces of $X$ parametrized by $h$ and linear operators $A_{h}: X_{h} \rightarrow X_{h}$.

We denote by $\sigma\left(A_{h}\right), \rho\left(A_{h}\right)$, and $R_{z}\left(A_{h}\right): X_{h} \rightarrow X_{h}$ the spectrum, resolvent set, and resolvent operator of $A_{h}$ considered as a bounded operator in $X_{h}$. However, when used in connection with expressions of the type " $\delta\left(A_{h}, A\right)$ " or " $\left\|A-A_{h}\right\|_{X_{h}}$ ", $A_{h}$ is considered a closed operator in $X$ with nondense domain $X_{h}$.

Let $\Gamma \subset \rho(A)$ be a given Jordan closed curve. Then

$$
E=\frac{1}{2 \pi i} \int_{\Gamma} R_{z}(A) d z: X \rightarrow X
$$

and, if $\Gamma \subset \rho\left(A_{h}\right)$,

$$
E_{h}=\frac{1}{2 \pi i} \int_{\Gamma} R_{z}\left(A_{h}\right) d z: X_{h} \rightarrow X_{h}
$$


are the spectral projectors relative to $A$ and $A_{h}$ respectively. We shall also use the relations

$$
A E=\frac{1}{2 \pi i} \int_{\Gamma} z R_{z}(A) d z: X \rightarrow X
$$

and

$$
A_{h} E_{h}=\frac{1}{2 \pi i} \int_{\Gamma} z R_{z}\left(A_{h}\right) d z: X_{h} \rightarrow X_{h}
$$

We now introduce the following two properties:

(P1) $\lim _{h \rightarrow 0} \delta\left(A_{h}, A\right) \rightarrow 0$,

(P2) $\forall x \in X, \lim _{h \rightarrow 0} \delta\left(x, X_{h}\right)=0$.

The following theorems contain the main results of this section. We note that $c<\infty$ shall denote, in this paper, a positive constant which is independent of $h$, but which varies from estimate to estimate.

TheOREM 1.1. Suppose (P1) is valid and let $K \subset \rho(A)$ be a compact set. Then there exists $h_{0}>0$ and $c=c(K)$ such that, for $h<h_{0}$, we have $K \subset \rho\left(A_{h}\right)$ and

$$
\left\|R_{z}(A)-R_{z}\left(A_{h}\right)\right\|_{X_{h}} \leqslant c \delta\left(A_{h}, A\right), \quad z \in K .
$$

Theorem 1.1 shows that if (P1) is valid, then the approximation of $\sigma(A)$ by $\sigma\left(A_{h}\right)$ is upper semicontinuous. Furthermore, for $h<h_{0},\left\|R_{z}\left(A_{h}\right)\right\|_{X_{h}}$ is uniformly bounded on $K$. This is a stability property.

THeOREM 1.2. Suppose (P1) is valid. Then there exists $h_{0}>0$ and $c$ so that, for $h<h_{0}$, we have the bounds

$$
\begin{gathered}
\left\|E-E_{h}\right\|_{X_{h}}+\left\|A E-A_{h} E_{h}\right\|_{X_{h}} \leqslant c \delta\left(A_{h}, A\right), \\
\delta\left(E_{h}\left(X_{h}\right), E(X)\right) \leqslant c \delta\left(A_{h}, A\right), \\
\delta\left(x, E_{h}\left(X_{h}\right)\right) \leqslant c\left\{\delta\left(x, X_{h}\right)+\delta\left(A_{h}, A\right)\|x\|\right\}, \quad x \in E(x) .
\end{gathered}
$$

THEOREM 1.3. Suppose that (P1) and (P2) are satisfied and that $E(X)$ is the finite-dimensional subspace corresponding to an isolated eigenvalue $\lambda$ of algebraic multiplicity $m$ of $A$. Let $\alpha$ be the ascent of $(\lambda-A)$ and let $f$ be a holomorphic function defined in the neighborhood of $\lambda$. We set

$$
\gamma_{h}=\min \left\{\delta\left(\left.A\right|_{E(X)}, A_{h}\right), \delta\left(A_{h}, A\right)\right\}
$$

Then, for $h$ small enough, $\left.A_{h}\right|_{E_{h}\left(X_{h}\right)}: E_{h}\left(X_{h}\right) \rightarrow E_{h}\left(X_{h}\right)$ has exactly $m$ eigenvalues $\lambda_{1, h}, \ldots, \lambda_{m, h}$, repeated according to multiplicity. Also, there exists $h_{0}>0$ and c such that the following bounds are valid for $h<h_{0}$ :

$$
\begin{array}{r}
\hat{\delta}\left(E_{h}\left(X_{h}\right), E(X)\right)<c \gamma_{h}, \\
\hat{\delta}\left(\left.A_{h}\right|_{E_{h}\left(X_{h}\right)},\left.A\right|_{E(X)}\right)<c \gamma_{h}, \\
\left|f(\lambda)-\frac{1}{m} \sum_{i=1}^{m} f\left(\lambda_{i, h}\right)\right|<c \gamma_{h}, \\
\max _{i=1, \ldots, m}\left|\lambda-\lambda_{i, h}\right|^{\alpha} \leqslant c \gamma_{h} .
\end{array}
$$


Remarks. If (P1) and (P2) are satisfied, then (1.3) of Theorem 1.2 shows that $\lim _{h \rightarrow 0} \delta\left(x, E_{h}\left(X_{h}\right)\right)=0, \forall x \in E(X)$, i.e., any $x \in E(X)$ can be approximated by vectors in $E_{h}\left(X_{h}\right)$. It is not true however that any $z \in \sigma(A)$ can be approximated by eigenvalues of $A_{h}$, i.e., there may exist $z \in \sigma(A)$ so that $\operatorname{dist}\left(z, \sigma\left(A_{h}\right)\right)$ fails to converge to zero. (The approximation of $\sigma(A)$ by $\sigma\left(A_{h}\right)$ is not necessarily lower semicontinuous.) The classical counterexample is the shift operator; see, for example, Kato [6, p. 210]. Note that if $A$ and $A_{h}$ are selfadjoint operators in a Hilbert space, then lower semicontinuity is true under weaker conditions than (P1), (P2); see, for example, Kato [6, p. 431].

In order to prove Theorems 1.1 and 1.2, we first prove a sequence of lemmas which are variants of results found in Kato [6, pp. 197-208].

Lemma 1.1. Let $B \in \mathscr{B}(X), C \in \mathcal{C}(X)$. Then

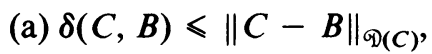

(b) $\|C-B\|_{\mathscr{D}(C)} \leqslant(1+\|B\|)^{2} \delta(C, B) /(1-(1+\|B\|) \delta(C, B))$

if the denominator is positive.

Proof. Part (a) follows directly from the definitions. We prove part (b). Let $x \in \mathscr{D}(C),\|x\|=1$, and let $\varepsilon>0$ be arbitrary. It follows from the definition of $\delta(C, B)$ that there exists $y \in X$ such that

$$
\|x-y\|+\|C x-B y\| \leqslant \delta(C, B)\{\|x\|+\|C x\|\}+\varepsilon .
$$

Consequently, we have

$$
\begin{aligned}
\|(C-B) x\| & \leqslant\|C x-B y\|+\|B y-B x\| \\
& \leqslant\|C x-B y\|+\|B\|\|y-x\| \\
& \leqslant(1+\|B\|)\{\|C x-B y\|+\|y-x\|\} \\
& \leqslant(1+\|B\|)[\delta(C, B)\{\|x\|+\|C x\|\}+\varepsilon] .
\end{aligned}
$$

Replacing $\|C x\|$ by $\|(C-B) x\|+\|B\|\|x\|$ in the right-hand side of the above inequality and letting $\varepsilon \rightarrow 0$ yields the estimate

$$
\|(C-B) x\| \leqslant(1+\|B\|) \delta(C, B)\{(1+\|B\|)\|x\|+\|(C-B) x\|\} .
$$

The result now follows from (1.10). Q.E.D.

Remark. It follows from Lemma 1.1 that if $A \in \mathscr{B}(X)$, then property (P1) is equivalent to $\lim _{h \rightarrow 0}\left\|A-A_{h}\right\|_{X_{h}}=0$. This is the spectral approximation condition of Descloux-Nassif-Rappaz [4].

Lemma 1.2. Let $B \in \mathscr{B}(X)$ and $C, D \in \mathcal{C}(X)$. Then

$$
\delta(C+B, D+B)<(1+\|B\|)^{2} \delta(C, D) .
$$

Proof. Let $x \in \mathscr{D}(C),\|x\|+\|(C+B) x\|=1$, and let $\varepsilon>0$ be arbitrary. We have then, by the triangle inequality, that

$$
\|x\|+\|C x\| \leqslant\|x\|+\|(C+B) x\|+\|B x\|<1+\|B\| .
$$

Thus, it follows from the definition of $\delta(C, D)$ that we may choose $y \in \mathscr{D}(D)$ such that

$$
\|x-y\|+\|C x-D y\|<\delta(C, D)(1+\|B\|)+\varepsilon .
$$


Hence,

$$
\begin{aligned}
\|x-y\|+\| & (C+B) x-(D+B) y \| \\
& \leqslant\|x-y\|+\|C x-D y\|+\|B\|\|x-y\| \\
& \leqslant(1+\|B\|)^{2} \delta(C, D)+(1+\|B\|) \varepsilon .
\end{aligned}
$$

Hence, the result follows from (1.13) after letting $\varepsilon \rightarrow 0$. Q.E.D.

Lemma 1.3. If $C$ and $D \in \mathcal{C}(X)$ are invertible, then

$$
\delta(C, D)=\delta\left(C^{-1}, D^{-1}\right) \text {. }
$$

Proof. This result follows directly from the definitions. Q.E.D.

LeMmA 1.4. Let $C, D \in \mathcal{C}(X)$ and suppose there exists $\kappa$ such that

$$
\|D x\| \geqslant \kappa\|x\|, \quad x \in \mathscr{D}(D) \text {. }
$$

If $\delta(C, D)<\min (1, \kappa)$, then $C$ is invertible.

Proof. We show that if $C$ is not invertible, then $\delta(C, D)>\min (1, \kappa)$. Let $x \in \mathscr{D}(C)$ be such that $\|x\|=1$ and $C x=0$. Let $\varepsilon>0$ be arbitrary. It follows from the definition of $\delta(C, D)$ that since $C x=0$ we can choose $y \in \mathscr{D}(D)$ such that

$$
\|x-y\|+\|D y\| \leqslant \delta(C, D)+\varepsilon .
$$

It then follows from (1.14) that

$$
\begin{aligned}
\delta(C, D)+\varepsilon & \geqslant\|x-y\|+\kappa\|y\| \geqslant \mid 1-\|y\|+\kappa\|y\| \\
& \geqslant \min (1, \kappa)\{|1-\|y\||+\|y\|\} \geqslant \min (1, \kappa) .
\end{aligned}
$$

Since $\varepsilon>0$ was arbitrary, it follows that we have reached the contradiction $\delta(C, D) \geqslant \min (1, \kappa)$. Q.E.D.

Proof of Theorem 1.1. In this proof, $c$ depends on $K$ but all estimates are uniform for $z \in K$. By Lemma 1.2,

$$
\delta\left(z-A_{h}, z-A\right) \leqslant c \delta\left(A_{h}, A\right), \quad z \in K .
$$

It follows from (1.16) and (P1) that

$$
\lim _{h \rightarrow 0} \delta\left(z-A_{h}, z-A\right) \rightarrow 0 \text { uniformly for } z \in K .
$$

Since $K \subset \rho(A)$, there exists $c_{1}$ such that

$$
\|(z-A) x\| \geqslant c_{1}\|x\|, \quad x \in \mathscr{D}(A), z \in K .
$$

We can now conclude from (1.17), (1.18) and Lemma 1.4 that there exists $h_{0}>0$ such that $z-A_{h}$ is invertible for $h<h_{0}$ and $z \in K$. Since $X_{h}$ is finite dimensional, we have that $K \subset \rho\left(A_{h}\right)$ for $h<h_{0}$.

Furthermore, it follows from (1.16) and Lemma 1.3 that

$$
\delta\left(R_{z}\left(A_{h}\right), R_{z}(A)\right) \leqslant c \delta\left(A_{h}, A\right), \quad z \in K .
$$

Hence, we can obtain from Lemma $1.1 \mathrm{~b}$ the result

$$
\begin{aligned}
\left\|R_{z}(A)-R_{z}\left(A_{h}\right)\right\|_{X_{h}} & \leqslant c \delta\left(R_{z}\left(A_{h}\right), R_{z}(A)\right) \\
& \leqslant c \delta\left(A_{h}, A\right), \quad h<h_{0}, z \in K . \quad \text { Q.E.D. }
\end{aligned}
$$


Proof of Theorem 1.2. The result (1.1) follows from Theorem 1.1 and the following estimates:

$$
\begin{aligned}
\| E- & E_{h}\left\|_{X_{h}}+\right\| A E-A_{h} E_{h} \|_{X_{h}} \\
& =\left\|\frac{1}{2 \pi i} \int_{\Gamma}\left(R_{z}(A)-R_{z}\left(A_{h}\right)\right) d z\right\|_{X_{h}}+\left\|\frac{1}{2 \pi i} \int_{\Gamma}\left(z R_{z}(A)-z R_{z}\left(A_{h}\right)\right) d z\right\|_{X_{h}} \\
& \leqslant(2 \pi)^{-1} \max _{z \in \Gamma}\left\|R_{z}(A)-R_{z}\left(A_{h}\right)\right\|_{X_{h}} \int_{\Gamma}(1+|z|)|d z| .
\end{aligned}
$$

The estimate (1.2) now follows directly from the estimate (1.1).

In order to prove (1.3), let $x \in E(X)$ and $x_{h} \in X_{h}$. Then $x-E_{h} x_{h}=E\left(x-x_{h}\right)$ $+\left(E-E_{h}\right) x_{h}$. Consequently,

$$
\begin{aligned}
\delta\left(x, E_{h}\left(X_{h}\right)\right) & \leqslant\left(\|E\|\left\|x-x_{h}\right\|+\left\|E-E_{h}\right\|_{x_{h}}\left\|x_{h}\right\|\right) \\
& \leqslant\left(\|E\|+\left\|E-E_{h}\right\|_{X_{h}}\right)\left\|x-x_{h}\right\|+\left\|E-E_{h}\right\|_{x_{h}}\|x\| .
\end{aligned}
$$

The result (1.3) follows by taking the infimum over $x_{h} \in X_{h}$ and using (1.1). Q.E.D.

It remains to prove Theorem 1.3. We first quote without proof the following simple result:

LEMmA 1.5. Let $Y$ and $Z$ be two subspaces of $X$ with the same finite dimension and let $P: Y \rightarrow Z$ be a linear operator such that

$$
\|P y-y\| \leqslant \frac{1}{2}\|y\|, \quad y \in Y .
$$

Then $P$ is bijective and

$$
\left\|P^{-1} z\right\| \leqslant 2\|z\|, \quad z \in Z .
$$

LEMMA 1.6. Let $Y$ and $Z$ be two subspaces of $X$.

(a) If $\hat{\delta}(Y, Z)<1$, then $\operatorname{dim} Y=\operatorname{dim} Z$.

(b) If $\operatorname{dim} Y=\operatorname{dim} Z<\infty$, then

$$
\delta(Y, Z) \leqslant \delta(Z, Y)[1-\delta(Z, Y)]^{-1} .
$$

Proof. For (a), see Kato [6, p. 200]. For (b), see Kato [7]. Q.E.D.

In the rest of this section, we suppose that the hypotheses of Theorem 1.3 are satisfied. Also, $c$ and $h_{0}$ will denote two generic positive constants which depend on $\Gamma$.

LeMma 1.7. $\delta\left(\left.A\right|_{E(X)},\left.A_{h}\right|_{E_{h}\left(X_{h}\right)}\right) \leqslant c \delta\left(\left.A\right|_{E(X)}, A_{h}\right), h \leqslant h_{0}$.

Proof. Let $x \in E(X),\|x\|+\|A x\|=1$, and let $x_{h} \in X_{h}$ be such that

$$
\left\|x-x_{h}\right\|+\left\|A x-A_{h} x_{h}\right\|<\delta\left(\left.A\right|_{E(X)}, A_{h}\right) \text {. }
$$

We have

$$
\begin{aligned}
\left\|x-E_{h} x_{h}\right\|+\| A x & -A_{h} E_{h} x_{h}\|=\| \frac{1}{2 \pi i} \int_{\Gamma}\left(R_{z}(A) x-R_{z}\left(A_{h}\right) x_{h}\right) d z \| \\
& +\left\|\frac{1}{2 \pi i} \int_{\Gamma}\left(z R_{z}(A) x-z R_{z}\left(A_{h}\right) x_{h}\right) d z\right\| \\
\leqslant(2 \pi)^{-1} & \max _{z \in \Gamma}\left\|R_{z}(A) x-R_{z}\left(A_{h}\right) x_{h}\right\| \int_{\Gamma}(1+|z|)|d z| .
\end{aligned}
$$


In order to conclude the proof of the lemma, it suffices to estimate $\left\|R_{z}(A) x-R_{z}\left(A_{h}\right) x_{h}\right\|$ for $z \in \Gamma$.

For $r_{h} \in X_{h}$, we have by Theorem 1 that

$$
\begin{aligned}
\| R_{z}(A) x & -R_{z}\left(A_{h}\right) x_{h}\|\leqslant\| R_{z}(A) x-r_{h}\|+\| r_{h}-R_{z}\left(A_{h}\right) x_{h} \| \\
& =\left\|R_{z}(A) x-r_{h}\right\|+\left\|R_{z}\left(A_{h}\right)\left[\left(z-A_{h}\right) r_{h}-x_{h}\right]\right\| \\
& \leqslant\left\|R_{z}(A) x-r_{h}\right\|+c\left\|\left(z-A_{h}\right) r_{h}-x_{h}\right\| \\
& \leqslant\left\|R_{z}(A) x-r_{h}\right\|+c\left(\left\|\left(z-A_{h}\right) r_{h}-(z-A) R_{z}(A) x\right\|+\left\|x-x_{h}\right\|\right) \\
& \leqslant c\left\|R_{z}(A) x-r_{h}\right\|+c\left\|A R_{z}(A) x-A_{h} r_{h}\right\|+c\left\|x-x_{h}\right\| .
\end{aligned}
$$

Since $R_{z}(A) x \in E(X)$, we can choose $r_{h} \in X_{h}$ so that

$$
\begin{aligned}
\| R_{z}(A) x & -r_{h}\|+\| A R_{z}(A) x-A_{h} r_{h} \| \\
& \leqslant \delta\left(\left.A\right|_{E(X)}, A_{h}\right)\left(\left\|R_{z}(A) x\right\|+\left\|A R_{z}(A) x\right\|\right) \\
& \leqslant c \delta\left(\left.A\right|_{E(X)}, A_{h}\right)(\|x\|+\|A x\|) \leqslant c \delta\left(\left.A\right|_{E(X)}, A_{h}\right) .
\end{aligned}
$$

The proof now follows by utilizing (1.26) and (1.29) in (1.28). Q.E.D.

Remark. Lemma 1.7 is still valid if the hypothesis $(\mathrm{P} 1)$ is replaced by the uniform boundedness of $R_{z}\left(A_{h}\right)$ on $\Gamma$.

Proof of Theorem 1.3. From (1.2) and (1.3) of Theorem 1.2, it follows that $\lim _{h \rightarrow 0} \hat{\delta}\left(E_{h}\left(X_{h}\right), E(X)\right)=0$. Consequently, by Lemma 1.6(a), $\operatorname{dim} E_{h}\left(X_{h}\right)=$ $\operatorname{dim} E(X)=m$ for $h<h_{0}$. This proves that for $h<h_{0},\left.A_{h}\right|_{E_{h}\left(X_{h}\right)}: E_{h}\left(X_{h}\right) \rightarrow E_{h}\left(X_{h}\right)$ has exactly $m$ eigenvalues $\lambda_{1, h}, \ldots, \lambda_{m, h}$ repeated according to multiplicity.

By Lemma 1.6(b), we have that

(1.30) $\hat{\delta}\left(E(X), E_{h}\left(X_{h}\right)\right) \leqslant c \min \left\{\delta\left(E(X), E_{h}\left(X_{h}\right)\right), \delta\left(E_{h}\left(X_{h}\right), E(X)\right)\right\}, \quad h<h_{0}$. It follows from Lemma 1.7 that

$$
\delta\left(E(X), E_{h}\left(X_{h}\right)\right) \leqslant c \delta\left(\left.A\right|_{E(X)},\left.A_{h}\right|_{E_{h}\left(X_{h}\right)}\right) \leqslant c \delta\left(\left.A\right|_{E(X)}, A_{h}\right) .
$$

The result (1.31) and (1.2) of Theorem 1.2 yield (1.4) when substituted in (1.30).

We have by (1.1) of Theorem 1.2 that

$$
\delta\left(\left.A_{h}\right|_{E_{h}\left(X_{h}\right)},\left.A\right|_{E(X)}\right) \leqslant c \delta\left(A_{h}, A\right), \quad h<h_{0} .
$$

Hypothesis (P1) and Lemmas 1.6 and 1.7 allow us to conclude the validity of (1.5).

Now let $u_{1}, \ldots, u_{m}$ be a basis for $E(X)$ with $\left\|u_{i}\right\|+\left\|A u_{i}\right\|=1, i=1, \ldots, m$. We then choose $u_{1, h}, \ldots, u_{m, h} \in E_{h}\left(X_{h}\right)$ so that

$$
\left\|u_{i}-u_{i, h}\right\|+\left\|A u_{i}-A_{h} u_{i, h}\right\| \leqslant \delta\left(\left.A\right|_{E(X)},\left.A_{h}\right|_{E_{h}\left(X_{h}\right)}\right), \quad i=1, \ldots, m .
$$

Next, we define $\Lambda_{h}: E(X) \rightarrow E_{h}\left(X_{h}\right)$ as the linear operator such that $\Lambda_{h} u_{i}=u_{i, h}$, $i=1, \ldots, m$. It follows by $(1.5)$ that

$$
\left\|u-\Lambda_{h} u\right\|+\left\|A u-A_{h} \Lambda_{h} u\right\| \leqslant c \gamma_{h}\|u\|, \quad u \in E(X) .
$$

By (P1) $\lim _{h \rightarrow 0} \gamma_{h}=0$, so by Lemma 1.5 and (1.34) $\Lambda_{h}$ is a bijection whose inverse $\Lambda_{h}^{-1}$ is uniformly bounded for $h<h_{0}$. Let $\hat{A}=\left.A\right|_{E(X)}$ and $\hat{A}_{h}=\Lambda_{h}^{-1} A_{h} \Lambda_{h}$ : $E(X) \rightarrow E(X)$. For $u \in E(X)$, we have

$$
\begin{aligned}
\left\|\left(\hat{A}-\hat{A}_{h}\right) u\right\| & =\left\|\Lambda_{h}^{-1}\left(\Lambda_{h} A-A_{h} \Lambda_{h}\right) u\right\| \\
& \leqslant c\left\{\left\|\left(\Lambda_{h}-I\right) A u\right\|+\left\|A u-A_{h} \Lambda_{h} u\right\|\right\} \leqslant c \gamma_{h}\|u\|, \quad h<h_{0} .
\end{aligned}
$$


Consequently,

$$
\left\|\hat{A}-\hat{A}_{h}\right\|_{E(X)} \leqslant c \gamma_{h} .
$$

Now $\hat{A}_{h}$ has eigenvalues $\lambda_{1, h}, \ldots, \lambda_{m, h}$ and $\hat{A}$ has the eigenvalue $\lambda$ of algebraic multiplicity $m$. Also, $\alpha$ is the ascent of $(\lambda-\hat{A})$. We have reduced our problem to the matrix case. The results (1.6) and (1.7) of Theorem 1.3 now follow from the classical perturbation theory for finite-dimensional operators [17, pp. 80-81]. Q.E.D.

2. The Approximation of the Spectrum of a Selfadjoint Operator. In this section, we suppose that $X$ is a Hilbert space, $A: \mathscr{D}(A) \subset X \rightarrow X$ is selfadjoint, and $A_{h}$ : $X_{h} \rightarrow X_{h}$ is selfadjoint in $X_{h}$ for all $h$. If $I \subset \mathbf{R}$ is an interval (finite or infinite), $E_{I}$ : $X \rightarrow X$ will denote the spectral projector of $A$ relative to $I$, and $E_{h, I}: X_{h} \rightarrow X_{h}$ will denote the spectral projector of $A_{h}$ relative to $I$. We can prove the following theorems:

THEOREM 2.1. (P1) $\Leftrightarrow \forall$ closed intervals $I, J$, one of them bounded, $I \cap J=\varnothing$, we have

$$
\lim _{h \rightarrow 0}\left\|E_{I} E_{h, J}\right\|_{X_{h}}=0
$$

TheOREM 2.2. Suppose that (P1) is valid. Let $J \subset I$ where $J$ is a closed bounded interval and $I$ is an open interval. Then

$$
\lim _{h \rightarrow 0} \delta\left(E_{h, J}\left(X_{h}\right), E_{I}(X)\right)=0 .
$$

THEOREM 2.3. Suppose that (P1) and (P2) are valid.

(a) Let $I$ be an open interval and $x \in E_{I}(X)$. Then

$$
\lim _{h \rightarrow 0} \delta\left(x, E_{h, I}\left(X_{h}\right)\right)=0 \text {. }
$$

(b) If $\lambda \in \sigma(A)$, then

$$
\lim _{h \rightarrow 0} \operatorname{dist}\left(\lambda, \sigma\left(A_{h}\right)\right)=0 .
$$

Remark. Theorem 2.3(b) states that the approximation of $\sigma(A)$ by $\sigma\left(A_{h}\right)$ is lower semicontinuous.

Theorems 2.1, 2.2, and 2.3 have been proved in Section 3 of Descloux, Nassif, Rappaz [4] when $A$ is bounded (recall our remarks after Lemma 1.1). Our proof will reduce the unbounded case to the bounded case. We shall restrict ourself to the proof of Theorem 2.2. Theorems 2.1 and 2.3 can be obtained by similar arguments. Furthermore, for the sake of simplicity and without much loss of generality, we shall suppose that $\rho(A) \cap \mathbf{R} \neq \varnothing$.

Proof of Theorem 2.2. As mentioned above, we suppose there exists $a \in \mathbf{R}$ with $a \in \rho(A)$. We introduce the function $\phi(\lambda)=(a-\lambda)^{-1}$ and set $B=\phi(A)=R_{a}(A)$. By Theorem 1.1, $B_{h}=\phi\left(A_{h}\right)=R_{a}\left(A_{h}\right)$ is well defined for $h$ sufficiently small and

$$
\lim _{h \rightarrow 0}\left\|B-B_{h}\right\|_{X_{h}}=0 \text {. }
$$

For an interval $M, F_{M}: X \rightarrow X$ and $F_{h, M}: X_{h} \rightarrow X_{h}$ will denote, respectively, the spectral projectors of $B$ and $B_{h}$ relative to $M$. As a first case, suppose $a \notin I$ and set $K=\phi(J)$ and $L=\phi(I)$. Then $K$ and $L$ are, respectively, a compact and an open 
interval with $K \subset L$. From Theorem 4 of Descloux, Nassif, Rappaz [4], we can conclude that

$$
\lim _{h \rightarrow 0} \delta\left(F_{h, K}\left(X_{h}\right), F_{L}(X)\right)=0 .
$$

However, the result (2.1) follows from (2.2) since $F_{h, K}\left(X_{h}\right)=E_{h, J}\left(X_{h}\right)$ and $F_{L}(X)=$ $E_{I}(X)$.

The case $a \in I$ can be reduced to the preceding one by noting that we can find compact intervals $J 1, J 2$ and open intervals $I 1, I 2$ with the following properties (for $h$ sufficiently small):

$$
\begin{gathered}
a \notin I 1 \cup I 2, \\
J 1 \subset I 1, \quad J 2 \subset I 2, \\
E_{I}(X)=E_{I 1}(X) \oplus E_{I 2}(X), \\
E_{h, J}\left(X_{h}\right)=E_{h, J 1}\left(X_{h}\right) \oplus E_{h, J 2}\left(X_{h}\right) . \quad \text { Q.E.D. }
\end{gathered}
$$

3. Application to Scalar Elliptic Boundary Value Problems. We shall need the following notation to discuss the application of our theory to the approximation of the spectral properties of scalar elliptic boundary value problems. Denote by $\Omega$ an open set in $\mathbf{R}^{n}$ with a smooth boundary, $\partial \Omega$. As usual, we denote by $L^{2}(\Omega)$ the Hilbert space of square integrable, complex-valued functions with inner product and norm

$$
\langle F, G\rangle=\int_{\Omega} F \bar{G} d x, \quad\|F\|^{2}=\langle F, F\rangle .
$$

We denote by $H^{r}(\Omega)$ the space of complex-valued functions whose distribution derivatives of order less than or equal to $r, r$ a nonnegative integer, are in $L^{2}(\Omega)$ with norm

$$
\|F\|_{r}^{2}=\sum_{|\alpha|<r}\left\|D^{\alpha} F\right\|^{2}
$$

We wish to consider the spectral approximation of the operator

$$
\begin{aligned}
& L u=-\sum_{i, j=1}^{n} D_{i}\left(a_{i j} D_{j} u\right)+\sum_{i=1}^{n} a_{i} D_{i} u+a u, \quad x \in \Omega, \\
& \mathscr{B} u=\sum_{i, j=1}^{n} a_{i j} \nu_{i} D_{j} u=0, \quad x \in \partial \Omega,
\end{aligned}
$$

where we assume that $L$ is a uniformly, strongly elliptic operator with real-valued coefficients in $C^{\infty}(\bar{\Omega})$ and $\nu=\left(\nu_{1}, \ldots, \nu_{n}\right)$ is the unit exterior normal to $\partial \Omega$. We associate with $L$ the continuous, sesquilinear form on $H^{1}(\Omega) \times H^{1}(\Omega)$,

$$
B(\phi, \psi)=\sum_{i, j=1}^{n}\left\langle a_{i j} D_{j} \phi, D_{i} \psi\right\rangle+\sum_{i=1}^{n}\left\langle a_{i} D_{i} \phi, \psi\right\rangle+\langle a \phi, \psi\rangle .
$$

We may assume (by replacing $L$ by $L+\mu, \mu \in \mathbf{R}$ ) that, for some $b>0$,

$$
\operatorname{Re} B(\phi, \phi) \geqslant b\|\phi\|_{1}^{2}, \quad \phi \in H^{1}(\Omega) .
$$

Let $\delta_{h} \subset H^{1}(\Omega)$ be a family of finite-dimensional subspaces, parametrized by $h$, $0<h \leqslant 1$, with $r$ a positive integer and $c$ a positive constant, independent of $h$, 
such that for $1 \leqslant s \leqslant r+1, u \in H^{s}(\Omega)$, we have

$$
\inf _{\chi \in \delta_{h}}\left(\|u-\chi\|+h\|u-\chi\|_{1}\right)<c h^{s}\|u\|_{s} .
$$

Many finite element spaces are known to satisfy (3.6) [2].

We define the operator $L_{h}: \mathcal{S}_{h} \rightarrow \mathcal{S}_{h}$ by the relation

$$
B\left(U_{h}, W\right)=\left\langle L_{h} U_{h}, W\right\rangle, \quad W \in \delta_{h} .
$$

Note that $L_{h}$ is well defined since $\delta_{h}$ is finite dimensional. We shall study the approximation of the spectral properties of $L$ by the spectral properties of $L_{h}$.

Define the space

$$
H_{\mathscr{B}}^{2}(\Omega)=\left\{u \in H^{2}(\Omega) \mid \mathscr{B} u=0 \text { for } x \in \partial \Omega\right\} .
$$

We consider $L$ as a closed operator from $L^{2}(\Omega)$ to $L^{2}(\Omega)$, i.e., $X=L^{2}(\Omega)$, with domain $D(L)=H_{\mathscr{P}}^{2}(\Omega)$. Also, in the notation of Section 1 , we set $X_{h}=\mathcal{S}_{h}$. It follows from (3.6) that if $u \in C^{\infty}(\bar{\Omega})$, then

$$
\delta\left(u, \delta_{h}\right)=\inf _{\chi \in \delta_{h}}\|u-\chi\| \rightarrow 0 \quad \text { as } h \rightarrow 0 .
$$

Since $C^{\infty}(\bar{\Omega})$ is dense in $L^{2}(\Omega)$, it is clear that property (P2) is valid.

We now turn to the verification of (P1). To that end, let $P_{h}^{0}: L^{2}(\Omega) \rightarrow \delta_{h}$ be the $L^{2}(\Omega)$ projection,

$$
\left\langle P_{h}^{0} u-u, W\right\rangle=0, \quad W \in \mathcal{S}_{h},
$$

and let $P_{h}^{1}: H^{1}(\Omega) \rightarrow \mathcal{S}_{h}$ be the $H^{1}(\Omega)$ projection defined by

$$
B\left(P_{h}^{1} u-u, W\right)=0, \quad W \in \mathcal{S}_{h} .
$$

It follows from (3.5) that $P_{h}^{1}$ is well defined.

The following estimates for $P_{h}^{0}$ and $P_{h}^{1}$ are well known [2]. There exists $c<\infty$ such that, for $0 \leqslant s \leqslant r+1$ and $u \in H^{s}(\Omega)$,

$$
\left\|P_{h}^{0} u-u\right\| \leqslant c h^{s}\|u\|_{s}
$$

and such that, for $1 \leqslant s \leqslant r+1$ and $u \in H^{s}(\Omega)$,

$$
\left\|P_{h}^{1} u-u\right\|+h\left\|P_{h}^{1} u-u\right\|_{1} \leqslant c h^{s}\|u\|_{s} .
$$

We now verify that

$$
\delta\left(L_{h}, L\right) \rightarrow 0 \text {. }
$$

For $U_{h} \in \mathcal{S}_{h}$, let $u \in D(L)$ satisfy

$$
L u=L_{h} U_{h}
$$

It follows by elliptic regularity that

$$
\|u\|_{2}<c\left\|L_{h} U_{h}\right\| \text {. }
$$

Now it is easily verified that $U_{h}=P_{h}^{1} u$. Hence, by (3.11) and (3.14),

$$
\left\|u-U_{h}\right\|=\left\|u-P_{h}^{1} u\right\| \leqslant c h^{2}\|u\|_{2} \leqslant c h^{2}\left\|L_{h} U_{h}\right\| .
$$

Since $L u=L_{h} U_{h}$, we can conclude that for each $U_{h} \in \mathcal{S}_{h}$, there exists $u \in D(L)$ such that

$$
\left\|u-U_{h}\right\|+\left\|L u-L_{h} U_{h}\right\| \leqslant c^{2}\left(\left\|U_{h}\right\|+\left\|L_{h} U_{h}\right\|\right) .
$$

Hence, we can conclude that

$$
\delta\left(L_{h}, L\right) \leqslant c h^{2}
$$


It is well known that the spectrum of $L$ consists of isolated eigenvalues with finite-dimensional generalized eigenspaces. Let $\lambda \in \sigma(L)$ and let $E(X)$ be the generalized eigenspace corresponding to $\lambda$. Thus, $\operatorname{dim} E(X)<\infty$. We shall show that

$$
\delta\left(\left.L\right|_{E(X)}, L_{h}\right) \leqslant c h^{r+1}
$$

The result (3.17) will imply that the conclusions of Theorem 1.3 are valid with $\gamma_{h} \leqslant c h^{r+1}$.

If $u \in E(X)$, set $U_{h}=P_{h}^{1} u \in \mathcal{S}_{h}$. It follows from elliptic regularity that $E(X) \subset$ $C^{\infty}(\bar{\Omega})$. Hence, by (3.11),

$$
\left\|u-P_{h}^{1} u\right\| \leqslant c h^{r+1}\|u\|_{r+1}
$$

Also, note that if $u \in H^{2}(\Omega)$, then

$$
P_{h}^{0} L u=L_{h} P_{h}^{1} u \text {. }
$$

Thus, it follows from (3.10) that

$$
\left\|L u-L_{h} U_{h}\right\|=\left\|L u-L_{h} P_{h}^{1} u\right\|=\left\|L u-P_{h}^{0} L u\right\| \leqslant c h^{r+1}\|L u\|_{r+1} .
$$

Note that since $\operatorname{dim} E(X)<\infty$ and all norms on finite-dimensional spaces are equivalent, there exists a constant $c<\infty$ such that, for $u \in E(X)$,

$$
\|L u\|_{r+1} \leqslant c\|u\|, \quad\|u\|_{r+1} \leqslant c\|u\| .
$$

So, (3.17) follows from (3.18) and (3.20).

The results on eigenvalue approximation implied by (3.17) are not the optimal $O\left(h^{2 r}\right)$ results for this problem that have been obtained by other methods [1], [14] unless $r=1$. However, it can be shown that optimal $O\left(h^{2 r}\right)$ eigenvalue results for the problem can be derived from Theorem 1.3 for this problem if the approximation of $L$ by $L_{h}$ is analyzed in a space $X$ which is taken to be an appropriate negative order Sobolev space.

4. Application to the Linearized Shallow Water Equations. We turn now to the description and analysis of an approximation procedure for the spectral properties of the operator, $T$, associated with the linearized shallow water equations. Recall that

$$
\begin{gathered}
T(\zeta, \vec{u})=(-\nabla \cdot \vec{u},-\nabla \zeta-f \vec{u}-\omega R \vec{u}), \quad x \in \Omega, \\
\vec{u} \cdot \vec{n}=0, \quad x \in \partial \Omega, \\
\int_{\Omega} \zeta d x=0,
\end{gathered}
$$

where $\Omega \subseteq \mathbf{R}^{2}$ is a bounded, connected open set with smooth boundary, $\partial \Omega$, $\vec{u}=\left(u_{1}, u_{2}\right), R$ is the linear operator $R \vec{u}=\left(-u_{2}, u_{1}\right), \vec{n}$ is the exterior normal to $\partial \Omega$, and $f \geqslant 0$ and $\omega$ are real constants representing friction and Coriolis terms. We assume that $\partial \Omega$ has a finite number of connected components, $\left\{\partial \Omega_{i}\right\}_{i=0}^{s}$. Also, assume that the sets $\partial \Omega_{i}$ are smooth arcs in $\mathbf{R}^{2}$. 
We shall need the definitions of the following spaces of scalar-valued functions:

$$
\begin{aligned}
& L_{*}^{2}(\Omega)=\left\{w \in L^{2}(\Omega) \mid \int_{\Omega} w d x=0\right\}, \\
& H_{*}^{1}(\Omega)=H^{1}(\Omega) \cap L_{*}^{2}(\Omega), \\
& H_{c}^{1}(\Omega)=\left\{w \in H^{1}(\Omega) \mid w(x)=0 \text { for } x \in \partial \Omega_{0},\right. \text { and there exist constants, } \\
&\left.\qquad\left(c_{i}\right)_{i=1}^{s}, \text { such that } w(x)=c_{i} \text { for } x \in \partial \Omega_{i}, i=1, \ldots, s\right\} .
\end{aligned}
$$

For $k \geqslant 1$, set

$$
H_{*}^{k}(\Omega)=H_{*}^{1}(\Omega) \cap H^{k}(\Omega), \quad H_{c}^{k}(\Omega)=H_{c}^{1}(\Omega) \cap H^{k}(\Omega) .
$$

We give these subspaces of $L^{2}(\Omega)$ and $H^{1}(\Omega)$ the inner product and norms defined in (3.1) and (3.2).

We also define the following spaces of vector-valued functions:

$$
\begin{aligned}
L^{2}(\Omega)^{2} & =\left\{\vec{u}=\left(u_{1}, u_{2}\right) \mid u_{1}, u_{2} \in L^{2}(\Omega)\right\}, \\
H^{k}(\Omega)^{2} & =\left\{\vec{u}=\left(u_{1}, u_{2}\right) \mid u_{1}, u_{2} \in H^{k}(\Omega)\right\} \quad \text { for } k \geqslant 1 .
\end{aligned}
$$

With these spaces we associate the inner product and norms

$$
\begin{aligned}
\langle\vec{u}, \vec{v}\rangle & =\left\langle u_{1}, v_{1}\right\rangle+\left\langle u_{2}, v_{2}\right\rangle, \\
\|\vec{u}\|^{2} & =\langle\vec{u}, \vec{u}\rangle, \quad \text { for } \vec{u}=\left(u_{1}, u_{2}\right), \vec{v}=\left(v_{1}, v_{2}\right) \in L^{2}(\Omega)^{2}, \\
\|\vec{u}\|_{k}^{2} & =\left\|u_{1}\right\|_{k}^{2}+\left\|u_{2}\right\|_{k}^{2}, \quad \text { for } \vec{u}=\left(u_{1}, u_{2}\right) \in H^{k}(\Omega)^{2} .
\end{aligned}
$$

Finally, for functions $(\zeta, \vec{u}),(\xi, \vec{v}) \in L_{*}^{2}(\Omega) \times L^{2}(\Omega)^{2}$ we define the inner product

$$
\langle(\zeta, \vec{u}),(\xi, \vec{v})\rangle=\langle\zeta, \xi\rangle+\langle\vec{u}, \vec{v}\rangle
$$

and norm

$$
\|(\zeta, u)\|^{2}=\langle(\zeta, \vec{u}),(\zeta, \vec{u})\rangle,
$$

and for functions $(\zeta, \vec{u}) \in H_{*}^{1}(\Omega) \times L^{2}(\Omega)^{2}$ we define the norm

$$
\|(\zeta, u)\|_{X}^{2}=\|\zeta\|_{1}^{2}+\|\vec{u}\|^{2}
$$

If $\vec{u} \in L^{2}(\Omega)^{2}$, then there exist unique scalar functions $\phi \in H_{*}^{1}(\Omega)$ and $\psi \in$ $H_{c}^{1}(\Omega)$ such that

$$
\vec{u}=-\nabla \phi+R \nabla \psi
$$

The functions $\{\phi, \psi\}$ are known as Stokes-Helmholtz potentials for $\vec{u}$ [8], [15], and we define the functions $S \vec{u}=\phi, H \vec{u}=\psi$. It follows from elliptic regularity [10] that for $k \geqslant 0$, there exists $c=c(k), c<\infty$, such that

$$
\|S \vec{u}\|_{k+1} \leqslant c\|\vec{u}\|_{k}, \quad\|H \vec{u}\|_{k+1} \leqslant c\|\vec{u}\|_{k}, \quad \forall \vec{u} \in H^{k}(\Omega)^{2} .
$$

It is easily verified that

$$
\langle\nabla \phi, R \nabla \psi\rangle=0, \quad \phi \in H_{*}^{1}(\Omega), \psi \in H_{c}^{1}(\Omega) .
$$

Hence, (4.4) is an orthogonal direct sum decomposition.

Let $\left\{\mathfrak{T}_{h}\right\}, 0<h \leqslant 1$, be a family of finite-dimensional subspaces of $H^{1}(\Omega)$ parametrized by $h$. Set

$$
\begin{aligned}
& \mathscr{K}_{h}^{c}=\mathscr{K}_{h} \cap H_{c}^{1}(\Omega), \\
& \mathscr{K}_{h}^{*}=\mathscr{K}_{h} \cap H_{*}^{1}(\Omega) .
\end{aligned}
$$


We assume that there exists a positive constant $c$, independent of $h$, and a positive integer $r$ such that, for $1 \leqslant k \leqslant r+1$ and $w \in H_{*}^{k}(\Omega)$,

$$
\inf _{\chi \in \mathscr{N}_{h}^{*}}\left(\|w-\chi\|+h\|w-\chi\|_{1}\right)<c h^{k}\|w\|_{k},
$$

and, for $1 \leqslant k \leqslant r+1$ and $w \in H_{c}^{k}(\Omega)$,

$$
\inf _{\chi \in \mathscr{T}_{h}^{c}}\left(\|w-\chi\|+h\|w-\chi\|_{1}\right) \leqslant c h^{k}\|w\|_{k} .
$$

We also wish to assume that the spaces $\left\{\mathfrak{N}_{h}\right\}$ satisfy the "inverse property" that there exists a positive constant $c$, independent of $h$, such that

$$
\|x\|_{1} \leqslant c h^{-1}\|x\|_{0}, \quad \chi \in \Re_{h} .
$$

We shall use the spaces of vector-valued functions

$$
\Re_{h}=\left\{\vec{U} \mid \vec{U}=-\nabla \Phi+R \nabla \Psi, \Phi \in \Re_{h}^{*}, \Psi \in \Re_{h}^{c}\right\} .
$$

It follows from (4.5), (4.9), and (4.10) that, for $0 \leqslant k \leqslant r, \vec{u} \in H^{k}(\Omega)^{2}$,

$$
\inf _{\vec{\chi} \in \mathscr{T}_{h}}\|\vec{u}-\vec{\chi}\| \leqslant c h^{k}\|\vec{u}\|_{k} \text {. }
$$

We shall need the result that if $\Psi \in \mathfrak{N}_{h}^{c}$, then

$$
\nabla \Psi \in \Re_{h} .
$$

It is easy to see that (4.12) is valid if $\mathfrak{T}_{h}$ contains the constant functions.

In order to define our approximation procedure, we introduce the continuous, sesquilinear form $B(\cdot, \cdot)$ on $X \times X=\left[H_{*}^{1}(\Omega) \times L^{2}(\Omega)^{2}\right] \times\left[H_{*}^{1}(\Omega) \times L^{2}(\Omega)^{2}\right]$ by

$$
\begin{array}{r}
B((\zeta, \vec{u}),(\xi, \vec{v}))=\langle\vec{u}, \nabla \xi\rangle-\langle\nabla \zeta, \vec{v}\rangle-\langle f \vec{u}+\omega R \vec{u}, \vec{v}\rangle, \\
(\zeta, \vec{u}),(\xi, \vec{v}) \in H_{*}^{1}(\Omega) \times L^{2}(\Omega)^{2} .
\end{array}
$$

We regard $T$ in (4.1) as an unbounded, closed operator, $T: H_{*}^{1}(\Omega) \times L^{2}(\Omega)^{2} \rightarrow$ $H_{*}^{1}(\Omega) \times L^{2}(\Omega)^{2}$, with domain

$$
D(T)=\left\{(\zeta, \vec{u}) \mid \zeta \in H_{*}^{1}(\Omega), \vec{u} \in L^{2}(\Omega)^{2}, \nabla \cdot \vec{u} \in H^{1}(\Omega), \vec{u} \cdot \vec{n}=0 \text { on } \partial \Omega\right\} .
$$

Thus, if $(\zeta, \vec{u}) \in D(T)$,

$$
B((\zeta, \vec{u}),(\xi, \vec{v}))=\langle T(\zeta, \vec{u}),(\xi, \vec{v})\rangle, \quad(\xi, \vec{v}) \in H_{*}^{1}(\Omega) \times L^{2}(\Omega)^{2} .
$$

We now define $T_{h}: \mathfrak{K}_{h}^{*} \times \mathscr{K}_{h} \rightarrow \mathfrak{T}_{h}^{*} \times \Re_{h}$ by

$$
B((Z, \vec{U}),(Y, \vec{V}))=\left\langle T_{h}(Z, \vec{U}),(Y, \vec{V})\right\rangle, \quad(Y, \vec{V}) \in \mathfrak{T}_{h}^{*} \times \Re_{h}
$$

The operator $T_{h}$ is well defined since the spaces $\mathfrak{N}_{h}^{*} \times \Re_{h}$ are finite dimensional.

We now show that the spectral properties of $T_{h}$ approximate those of $T$ by verifying (P1) and (P2) in this case for $X=H_{*}^{1}(\Omega) \times L^{2}(\Omega)^{2}$ and $X_{h}=\mathfrak{T}_{h}^{*} \times \Re_{h}$. The verification of (P2) follows directly from (4.9) and (4.11). To see this, we note that for $(\zeta, \vec{u}) \in\left[L_{*}^{2}(\Omega) \cap C^{\infty}(\bar{\Omega})\right] \times C^{\infty}(\bar{\Omega})^{2}$ it follows from (4.9) and (4.11) that

$$
\delta\left((\zeta, \vec{u}), X_{h}\right) \rightarrow 0 \text { as } h \rightarrow 0 .
$$

The validity of (P2) now follows from the density of $\left[L_{*}^{2}(\Omega) \cap C^{\infty}(\bar{\Omega})\right] \times C^{\infty}(\bar{\Omega})^{2}$ in $H_{*}^{1}(\Omega) \times L^{2}(\Omega)^{2}$.

We next turn to the verification of (P1). In order to prove this result, we introduce and analyze some projection operators. First, we define the $H_{*}^{1}(\Omega)$ 
projection $P_{h}^{*}: H_{*}^{1}(\Omega) \rightarrow \mathscr{T}_{h}^{*}$ by

$$
\left\langle\nabla\left(P_{h}^{*} w-w\right), \nabla \chi\right\rangle=0, \quad \chi \in \Re_{h}^{*},
$$

and the $H_{c}^{1}(\Omega)$ projection $P_{h}^{c}: H_{c}^{1}(\Omega) \rightarrow \Re_{h}^{c}$ by

$$
\left\langle\nabla\left(P_{h}^{c} w-w\right), \nabla \chi\right\rangle=0, \quad \chi \in \mathfrak{N}_{h}^{c} .
$$

It is well known [2] that there exists $c<\infty$ such that, for $1<k<r+1$ and $w \in H_{*}^{k}(\Omega)$,

$$
\left\|P_{h}^{*} w-w\right\|_{1} \leqslant c h^{k-1}\|w\|_{k}
$$

and, for $1 \leqslant k \leqslant r+1$ and $w \in H_{c}^{k}(\Omega)$,

$$
\left\|P_{h}^{c} w-w\right\|_{1} \leqslant c h^{k-1}\|w\|_{k} .
$$

It follows from (4.11) that if $\vec{Q}_{h}: L^{2}(\Omega)^{2} \rightarrow \Re_{h}$ is the $L^{2}(\Omega)^{2}$ projection defined by

$$
\left\langle\vec{Q}_{h} \vec{u}-\vec{u}, \vec{\chi}\right\rangle=0, \quad \vec{\chi} \in \Re_{h},
$$

then there exists a constant $c<\infty$ such that $0<k<r$ and $\vec{u} \in H^{k}(\Omega)^{2}$ imply that

$$
\left\|\vec{Q}_{h} \vec{u}-\vec{u}\right\|<c h^{k}\|\vec{u}\|_{k}
$$

It is easily checked that

$$
\vec{Q}_{h} \vec{u}=-\nabla P_{h}^{*} S \vec{u}+R \nabla P_{h}^{c} H \vec{u} .
$$

Finally, define the $L^{2}(\Omega)$ projection $\hat{Q}_{h}: L_{*}^{2}(\Omega) \rightarrow \mathfrak{T}_{h}^{*}$ by

$$
\left\langle\hat{Q}_{h} w-w, \chi\right\rangle=0, \quad \chi \in \mathfrak{T}_{h}^{*} \text {. }
$$

It follows from (4.9) and the inverse property of $\mathfrak{T}_{h}$ that there exists $c<\infty$ such that if $1 \leqslant k \leqslant r+1$ and $w \in H_{*}^{k}(\Omega)$, then

$$
\left\|\hat{Q}_{h} w-w\right\|_{1} \leqslant c h^{k-1}\|w\|_{k} \text {. }
$$

The following lemmas will help us analyze $T_{h}$.

Lemma 4.1. (a) Let $\vec{u} \in L^{2}(\Omega)^{2}$ and $\chi \in \mathfrak{T}_{h}^{*}$. Then

$$
\left\langle\vec{u}-\vec{Q}_{h} \vec{u}, \nabla \chi\right\rangle=0 .
$$

(b) Let $\zeta \in H_{*}^{1}(\Omega)$ and $\vec{\chi} \in \Re_{h}$. Then

$$
\left\langle\nabla\left(\zeta-P_{h}^{*} \zeta\right), \vec{\chi}\right\rangle=0 .
$$

Proof. The proof follows easily from (4.6) and the definitions of $P_{h}^{*}$ and $\vec{Q}_{h}$. Q.E.D.

Now let $\mathscr{P}_{h}=P_{h}^{*} \oplus \vec{Q}_{h}: H_{*}^{1}(\Omega) \times L^{2}(\Omega)^{2} \rightarrow \mathscr{N}_{h}^{*} \times \mathscr{K}_{h}$.

LEMMA 4.2. If $(\zeta, \vec{u}) \in D(T)$ and $(Y, \vec{V}) \in \Re_{h}^{*} \times \Re_{h}$, then

$$
\begin{aligned}
B\left((\zeta, \vec{u})-\mathscr{P}_{h}(\zeta, \vec{u}),(Y, \vec{V})\right) & =\left\langle T(\zeta, \vec{u})-T_{h} \mathscr{P}_{h}(\zeta, \vec{u}),(Y, \vec{V})\right\rangle \\
& =-\left\langle\omega R\left(u-\vec{Q}_{h} \vec{u}\right), \vec{V}\right\rangle .
\end{aligned}
$$

Proof. The proof follows directly from the definition of $B$ and Lemma 4.1. Q.E.D.

Hence, it follows from Lemma 4.2 that if $Q_{h}=\hat{Q}_{h} \oplus \vec{Q}_{h},(\zeta, \vec{u}) \in D(T)$, and $(Y, \vec{V}) \in \mathfrak{N}_{h}^{*} \times \Re_{h}$, then

$$
\left\langle Q_{h} T(\zeta, \vec{u})-T_{h} \mathscr{P}_{h}(\zeta, \vec{u}),(Y, \vec{V})\right\rangle=-\left\langle\omega R\left(\vec{u}-\vec{Q}_{h} \vec{u}\right), \vec{V}\right\rangle .
$$


THEOREM 4.1. $\delta\left(T_{h}, T\right) \leqslant c h$.

Proof. Let $(Z, \vec{U}) \in \mathfrak{N}_{h}^{*} \times \Re_{h}$ and denote $T_{h}(Z, \vec{U})$ by $(D, \vec{E})$. We must find $(\zeta, \vec{u}) \in D(T)$ such that

$$
\begin{aligned}
\|(Z, \vec{U})-(\zeta, \vec{u})\|_{X}+ & \left\|T_{h}(Z, \vec{U})-T(\zeta, \vec{u})\right\|_{X} \\
& \leqslant \operatorname{ch}\left(\|(Z, \vec{U})\|_{X}+\left\|T_{h}(Z, \vec{U})\right\|_{X}\right) .
\end{aligned}
$$

We choose $(\zeta, \vec{u})$ as follows. Let $\phi \in H_{*}^{2}(\Omega)$ be the solution to

$$
\begin{aligned}
& \Delta \phi=D, \quad x \in \Omega, \\
& \frac{\partial \phi}{\partial n}=0, \quad x \in \partial \Omega,
\end{aligned}
$$

where $\partial / \partial n$ is the exterior normal derivative. (Note that $\int_{\Omega} D d x=0$ since $D \in$ $L_{*}^{2}(\Omega)$.) Now set $\zeta=Z, \vec{u}=-\nabla \phi+R \nabla H \vec{U}$. It is clear that since $Z \in H_{*}^{1}(\Omega)$, $\nabla \cdot \vec{u}=-\Delta \phi=-D \in H^{1}(\Omega), \vec{u} \cdot \vec{n}=-\partial \phi / \partial n=0$ for $x \in \partial \Omega$ that $(\zeta, \vec{u}) \in D(T)$.

It follows by the definition of $\phi$ that $P_{h}^{*} \phi=S \vec{U}$. By elliptic regularity for (4.31),

$$
\|\phi\|_{2} \leqslant c\|D\| \leqslant c\|D\|_{1} \text {. }
$$

Hence, we can conclude from (4.19) that

$$
\|\nabla(S \vec{u}-S \vec{U})\|=\left\|\nabla\left(\phi-P_{h}^{*} \phi\right)\right\|<\operatorname{ch}\|\phi\|_{2} \leqslant \operatorname{ch}\|D\|_{1} .
$$

So,

$$
\begin{aligned}
\|(Z, \vec{U})-(\zeta, \vec{u})\|_{X} & =\|\nabla(S \vec{u}-S \vec{U})\| \leqslant c h\|D\|_{1} \\
& \leqslant c h\left\|T_{h}(Z, \vec{U})\right\|_{X} .
\end{aligned}
$$

Denote $T(\zeta, \vec{u})$ by $(d, \vec{e})$. It follows from (4.31) that $d=D$. Since $\mathscr{P}_{h}(\zeta, \vec{u})=$ $(Z, \vec{U})$, we obtain from (4.29)

$$
\left\langle\vec{Q}_{h} \vec{e}-\vec{E}, \vec{V}\right\rangle=\left\langle\omega R\left(\nabla \phi-\nabla P_{h}^{*} \phi\right), \vec{V}\right\rangle
$$

Thus, if we set $\vec{V}=\vec{Q}_{h} \vec{e}-\vec{E}$ in (4.34) and use the Cauchy-Schwarz inequality, we can derive the estimate

$$
\begin{aligned}
\left\|\vec{Q}_{h} \vec{e}-\vec{E}\right\| & \leqslant c\left\|\nabla \phi-\nabla P_{h}^{*} \phi\right\| \leqslant c h\|\phi\|_{2} \\
& \leqslant c h\|D\|_{1} \leqslant c h\left\|T_{h}(Z, \vec{U})\right\|_{X} .
\end{aligned}
$$

We must now estimate

$$
\left\|\vec{e}-\vec{Q}_{h} \vec{e}\right\|=\inf _{\vec{\chi} \in \Re_{h}}\|\vec{e}-\chi\|
$$

By the definition of $T$,

(4.37) $\vec{e}=-\nabla \zeta-f \vec{u}-\omega R \vec{u}=-\nabla Z-f(-\nabla \phi+R \nabla H \vec{U})-\omega(-R \nabla \phi-\nabla H \vec{U})$.

However,

$$
-\nabla Z-f R \nabla H \vec{U}+\omega \nabla H \vec{U} \in \Re_{h}
$$

and

$$
\|f \nabla \phi+\omega R \nabla \phi\|_{1} \leqslant c\|D\|_{1}
$$

So,

$$
\begin{aligned}
\left\|\vec{e}-\vec{Q}_{h} \vec{e}\right\| & =\inf _{\vec{\chi} \in \mathscr{T}_{h}}\|f \nabla \phi+\omega R \nabla \phi-\vec{\chi}\|<\operatorname{ch}\|f \nabla \phi+\omega R \nabla \phi\|_{1} \\
& \leqslant \operatorname{ch}\|D\|_{1} \leqslant \operatorname{ch}\left\|T_{h}(Z, \vec{U})\right\|_{X} .
\end{aligned}
$$


The triangle inequality, (4.35), and (4.40) yield

$$
\left\|T(\zeta, \vec{u})-T_{h}(Z, \vec{U})\right\|_{X}=\|\vec{e}-\vec{E}\| \leqslant \operatorname{ch}\left\|T_{h}(Z, \vec{U})\right\|_{X} .
$$

Thus, (4.30) follows from (4.33) and (4.41). Q.E.D.

Now let $\lambda \in \sigma(T)$ be an isolated eigenvalue of $T$ with finite algebraic multiplicity $m$. Let $E(X)$ be the generalized eigenspace corresponding to $\lambda$. It follows from Theorem 1.3 that we are interested in an estimate for $\delta\left(\left.T\right|_{E(X)}, T_{h}\right)$.

TheOrem 4.2. Let $\lambda \in \sigma(T)$ be an isolated eigenvalue of $T$ and let $E(X)$ be its associated generalized eigenspace. Assume $\operatorname{dim} E(X)=m<\infty$, and assume that $(\zeta, \vec{u}) \in E(X)$ implies that $\zeta \in H_{*}^{r+1}(\Omega), \vec{u} \in H^{r}(\Omega)^{2}, \nabla \cdot \vec{u} \in H^{r}(\Omega)$. Then there exists $c<\infty$, independent of $h$, such that

$$
\delta\left(\left.T\right|_{E(X)}, T_{h}\right) \leqslant c h^{r} .
$$

Proof. Let $(\zeta, \vec{u}) \in E(X)$. Set $(Z, \vec{U})=\mathscr{P}_{h}(\zeta, \vec{u})$. It follows from (4.19) and (4.22) that

$$
\left\|(\zeta, \vec{u})-\mathscr{P}_{h}(\zeta, \vec{u})\right\|_{X} \leqslant \operatorname{ch}^{r}\left(\|\zeta\|_{r+1}+\|\vec{u}\|_{r}\right) .
$$

Also, setting $(Y, \vec{V})=Q_{h} T(\zeta, \vec{u})-T_{h} \mathscr{P}_{h}(\zeta, \vec{u})$ in (4.29) and using the CauchySchwarz inequality, we obtain

$$
\begin{aligned}
\left\|Q_{h} T(\zeta, \vec{u})-T_{h} \mathscr{P}_{h}(\zeta, \vec{u})\right\|_{X} & =\left\|Q_{h} T(\zeta, \vec{u})-T_{h} \mathscr{P}_{h}(\zeta, \vec{u})\right\| \\
& \leqslant c\left\|\vec{u}-\vec{Q}_{h} \vec{u}\right\| \leqslant c h^{r}\|\vec{u}\|_{r} .
\end{aligned}
$$

Finally, from (4.22) and (4.24) we obtain

$$
\left\|T(\zeta, \vec{u})-Q_{h} T(\zeta, \vec{u})\right\|_{X} \leqslant \operatorname{ch}^{r}\left(\|\zeta\|_{r+1}+\|\vec{u}\|_{r}+\|\nabla \cdot \vec{u}\|_{r}\right) .
$$

The result of the theorem now follows from (4.43), (4.44), (4.45), and the finite dimensionality of $E(X)$. Q.E.D.

Finally, we note that our results can be combined with the results in [3] to obtain optimal order estimates on the convergence of eigenvalues. Assume that the conditions of Theorem 4.2 are valid. Let $E\left(X^{*}\right)$ denote the generalized eigenspace associated with the eigenvalue $\bar{\lambda}$ of $T^{*}$. We note that it is well known that $\operatorname{dim} E\left(X^{*}\right)=m$ and that $\bar{\lambda}$ is an isolated eigenvalue.

Let

$$
\hat{w}_{h}=\delta\left(E(X), E\left(X_{h}\right)\right), \quad \hat{w}_{h}^{*}=\delta\left(E\left(X^{*}\right), E\left(X_{h}^{*}\right)\right) .
$$

Let $\alpha$ be the ascent of $\lambda$. Then it follows from Proposition 3.2 of [3] that for $h_{0}$ sufficiently small

$$
\max _{i=1, \ldots, m}\left|\lambda-\lambda_{i, h}\right|^{\alpha} \leqslant c \hat{w}_{h} \hat{w}_{h}^{*}, \quad\left|\lambda-\frac{1}{m} \sum_{i=1}^{m} \lambda_{i, h}\right| \leqslant c \hat{w}_{h} \hat{w}_{h}^{*}, \quad h \leqslant h_{0} .
$$

Under the hypotheses of Theorem 4.2 we have proven that $\hat{w}_{h}<c h^{r}$. Now assume, in addition, that $(\zeta, \vec{u}) \in E\left(X^{*}\right)$ implies that $\zeta \in H_{*}^{r+1}(\Omega), \vec{u} \in H^{r}(\Omega)^{2}, \nabla \cdot \vec{u} \in$ $H^{r}(\Omega)$. Then we can conclude, from applying the above arguments to the adjoint problem, that $\hat{w}_{h}^{*} \leqslant c h^{r}$. Hence, under the above conditions, we obtain the optimal order eigenvalue estimates

$$
\max _{i=1, \ldots, m}\left|\lambda-\lambda_{i, h}\right|^{\alpha} \leqslant c h^{2 r}, \quad\left|\lambda-\frac{1}{m} \sum_{i=1}^{m} \lambda_{i, h}\right|<c h^{2 r}, \quad h<h_{0} .
$$


Département de Mathématiques

École Polytechnique Fédérale

1007 Lausanne, Switzerland

Department of Mathematics

The University of Michigan

Ann Arbor, Michigan 48109

Centre de Mathématiques Appliquées

Ecole Polytechnique

91128 Palaiseau, France

1. J. H. Bramble \& J. E. Osborn, "Rate of convergence estimates for nonselfadjoint eigenvalue approximations," Math. Comp., v. 27, 1973, pp. 525-549.

2. P. G. Ciarlet, The Finite Element Method for Elliptic Problems, North-Holland, Amsterdam, 1978.

3. J. Descloux, "Error bounds for an isolated eigenvalue obtained by the Galerkin method," $Z$. Angew. Math. Phys., v. 30, 1979, pp. 167-176.

4. J. Descloux, N. Nassif \& J. Rappaz, "On spectral approximation, Part 1. The problem of convergence," R.A.I.R.O. Anal. Numér., v. 12, no. 2, 1978, pp. 97-112.

5. J. Descloux, N. Nassif \& J. RAPPAZ, "On spectral approximation, Part 2. Error estimates for the Galerkin method," R.A.I.R.O. Anal. Numér., v. 12, no. 2, 1978, pp. 113-119.

6. T. KAтo, Perturbation Theory for Linear Operators, Die Grundlehren der Math. Wissenschaften, Band 132, Springer-Verlag, New York, 1966.

7. T. KATo, "Perturbation theory for nullity, deficiency, and other quantities of linear operators," $J$. Analyse Math., v. 6, 1958, pp. 261-322.

8. H. Lamb, Hydrodynamics, 6th ed., Cambridge Univ. Press, Cambridge, 1932.

9. P. D. LAX \& R. PHLluPs, "Local boundary conditions for dissipative symmetric differential operators," Comm. Pure Appl. Math., v. 13, 1960, pp. 427-455.

10. J. L. Lions \& E. Magenes, Problemes aux Limites Non Homogenes et Applications, Vol. 1, Dunod, Paris, 1968.

11. M. LuSkIN, "Convergence of a finite element method for the approximation of normal modes of the oceans," Math. Comp., v. 33, 1979, pp. 493-519.

12. W. Mills, JR., "The resolvent stability condition for spectra convergence with application to the finite element approximation of noncompact operators," SIAM J. Numer. Anal., v. 16, 1979, pp. 695-703.

13. W. Mills, JR., "Optimal error estimates for the finite element spectral approximation of noncompact operators," SIAM J. Numer. Anal., v. 16, 1979, pp. 704-718.

14. J. E. OsBorN, "Spectral approximations for compact operators," Math. Comp., v. 29, 1975, pp. $712-725$.

15. G. W. Platzman, "Normal modes of the Atlantic and Indian Oceans," J. Phys. Oceanography, v. 5, 1975, pp. 201-221.

16. F. RIESz \& B. Sz.-NAGY, Functional Analysis, Ungar, New York, 1955.

17. J. H. Wilkinson, The Algebraic Eigenvalue Problem, Clarendon Press, Oxford, 1965. 